\title{
Clinical application of cone beam computed tomography for ideal absorbable membrane placement in interproximal bone defects
}

\author{
Masatoshi Takane ${ }^{1,2)}$, Shuichi Sato ${ }^{1,2)}$, Kuniharu Suzuki $^{1)}$, Takeshi Fukuda ${ }^{1)}$, \\ Yukihiro Asano ${ }^{1)}$, Kazuya Honda ${ }^{3,4)}$, Yoshinori Arai ${ }^{5)}$ and Koichi Ito ${ }^{1,2)}$ \\ ${ }^{1)}$ Department of Periodontology, Nihon University School of Dentistry, Tokyo, Japan \\ ${ }^{2)}$ Division of Advanced Dental Treatment, Dental Research Center, Nihon University School of Dentistry, \\ Tokyo, Japan \\ ${ }^{3)}$ Department of Radiology, Nihon University School of Dentistry, Tokyo, Japan \\ ${ }^{4)}$ Division of Oral and Craniomaxillofacial Research, Dental Research Center, \\ Nihon University School of Dentistry, Tokyo, Japan \\ ${ }^{5)}$ Nihon University School of Dentistry, Tokyo, Japan
}

(Received 23 July and accepted 10 December 2009)

\begin{abstract}
When a guided tissue regeneration (GTR) membrane is placed in an interproximal area, adjustment of the membrane is difficult because of the complex anatomy of the root surface and presence of bone defects, thus prolonging the surgery. This report describes the clinical application of cone beam computed tomography (CT) images to aid insertion of a barrier membrane in the treatment of interproximal bone defects. Using $\mathrm{CT}$ images, the membrane can be pre-cut into the optimal shape to cover the bone defect and fit the roots tightly, thus shortening the time required to adjust the GTR membrane, and providing excellent clinical outcomes. (J Oral Sci 52, 63-69, 2010)
\end{abstract}

Keywords: GTR therapy; intrabony defects; membrane design; cone beam CT; diagnosis.

Correspondence to Dr. Shuichi Sato, Department of Periodontology, Nihon University School of Dentistry, 1-8-13 Kanda Surugadai, Chiyoda-ku, Tokyo 101-8310, Japan

Tel: +81-3-3219-8107

Fax: +81-3-3219-8349

E-mail: sato-su@dent.nihon-u.ac.jp

\section{Introduction}

Evidence suggests that guided tissue regeneration (GTR) therapy with a barrier membrane can successfully treat intrabony defects (1-4).The purpose of placing materials over intrabony defects is to keep the gingival epithelium and connective tissue away from the root surface while bone is being regenerated. Currently, non-absorbable expanded polytetrafluoroethylene (e-PTFE) and absorbable membranes are used as barriers in GTR therapy. These barriers are preformed into various sizes and shapes to fit periodontal defects of different morphologies and in different locations. For GTR therapy to be successful, tight fitting of the membrane to the root surface and complete coverage of the bone defect are important (5). However, most membranes must be individually trimmed and adjusted to fit the root and cover the bone defect. In particular, when a GTR membrane is placed in an interproximal area, the bone defects complicate the adjustment of the GTR membrane, thus prolonging the operation.

Cone beam computed tomography (CT) is widely used for three-dimensional (3-D) maxillofacial imaging dentistry (6-10) and can be applied for diagnosis of periodontal bone defects (11). It can clearly visualize the morphology of bone defects and furcation involvement, and the data are easily 
converted into 3-D images. In the present study, we examined the use of cone beam CT images when using a barrier membrane in the treatment of intrabony defects.

\section{Basic Study}

\section{Materials and Methods}

Nine dentists (all with more than 5 years of clinical experience, and 6 being periodontists) practicing at the Department of Periodontology, Nihon University School of Dentistry, Tokyo, Japan, participated in this study. The periodontal disease model had a two-wall intrabony defect in the interproximal area between \#26 and \#27 (Fig. 1).

A periapical radiograph of this area was taken using a dental holder and scanned by cone beam CT (3DX Multi Image Micro CT, Morita Co. Kyoto, Japan). The reconstructed image was a cylinder $32 \mathrm{~mm}$ in height and

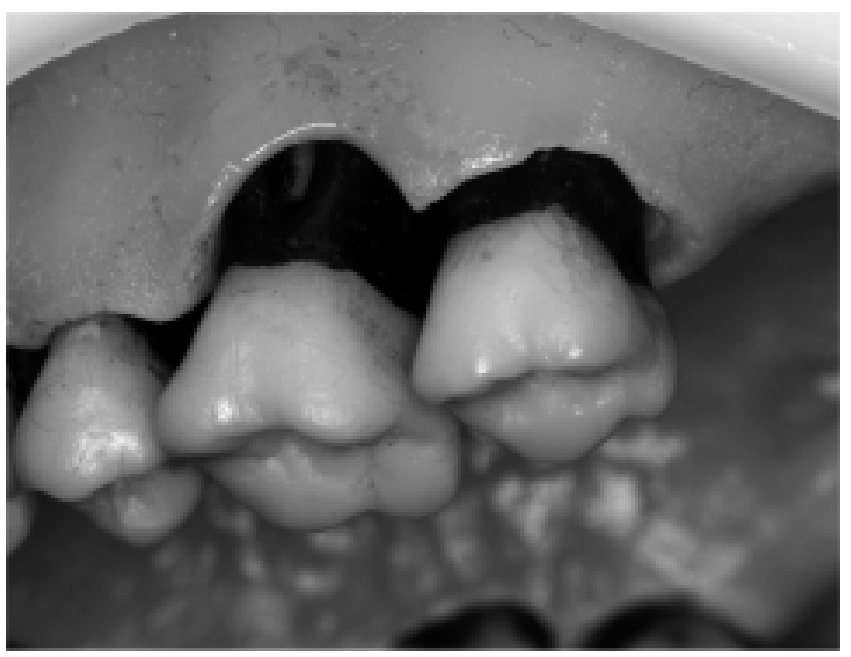

Fig. 1 Two-wall defect between \#26 and \#27.
$38 \mathrm{~mm}$ in diameter. The voxel size was a $0.136-\mathrm{mm}$ orthocube. Scanning was done at $85 \mathrm{kV}$ and $10 \mathrm{~mA}$, with a 1$\mathrm{mm}$ copper filter, for $17 \mathrm{~s}$. A single scan collected 512 sets of two-dimensional projection data through 360 degrees. The image was reconstructed with a personal computer within approximately $10 \mathrm{~min}$.

Each participant trimmed and cut a membrane to fit the defect (conventional method). An axial CT image based on a periapical radiograph was used to make the ideal fitted membrane. The outline of the membrane was drawn using computer software, and each participant cut a template (CT method) (Fig. 2).

To evaluate the adaptation of both membranes to the bone defect, we measured the areas of the membranes prepared using the CT method and the conventional method. The area was divided into interproximal (Fig. 3a) and buccal sites. The membrane adaptation of the interproximal sites was rated fit or not fit, and the buccal site was rated according to whether over $3 \mathrm{~mm}$ of the defect was covered by the membrane (Fig. 3b). In addition, we compared the membrane trimming and adjustment times between the $\mathrm{CT}$ method and the conventional method.

\section{Statistical analysis}

All data are presented as mean \pm SD. The data were analyzed using Student's $t$-test at a significance level of $P<0.05$. The SPSS software package was used for all analyses.

\section{Results}

The membrane in the interproximal area was significantly larger when the CT method was used, as compared with the conventional method (Figs. 4 and 5), and the mesial
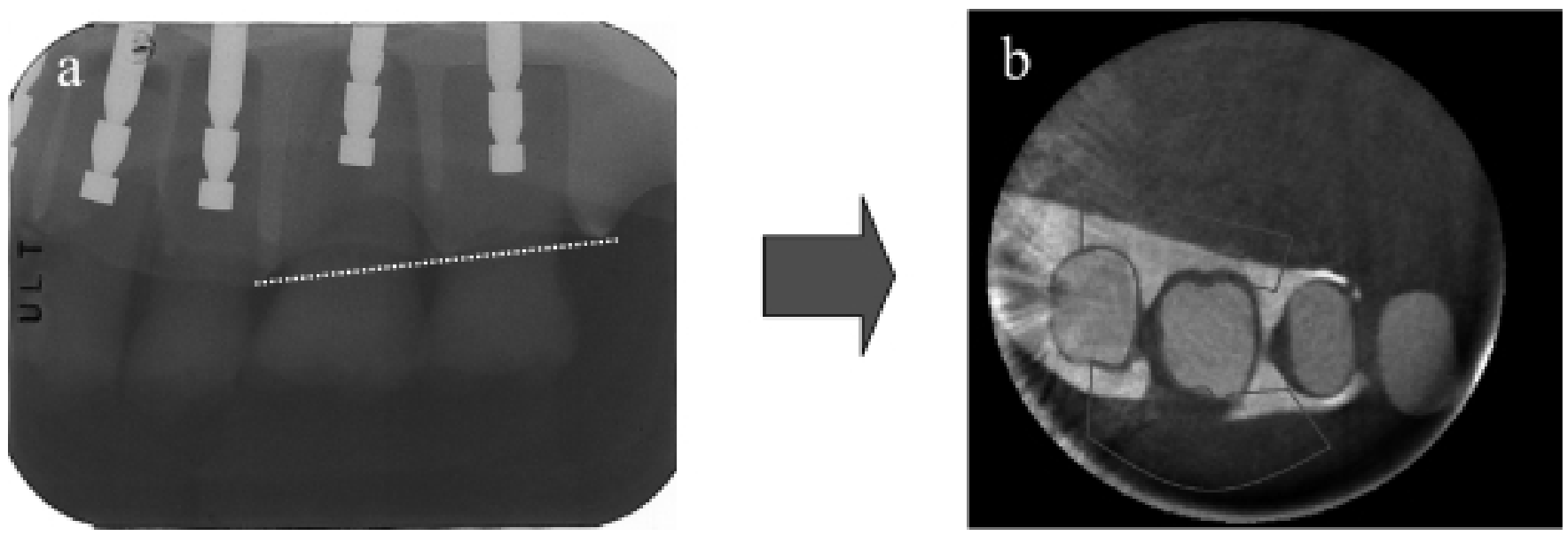

Fig. 2 (a) Periapical radiograph. (b) Horizontal CT images obtained in the plane of the periapical radiograph (dotted line). The solid line shows the outline of the membrane. 

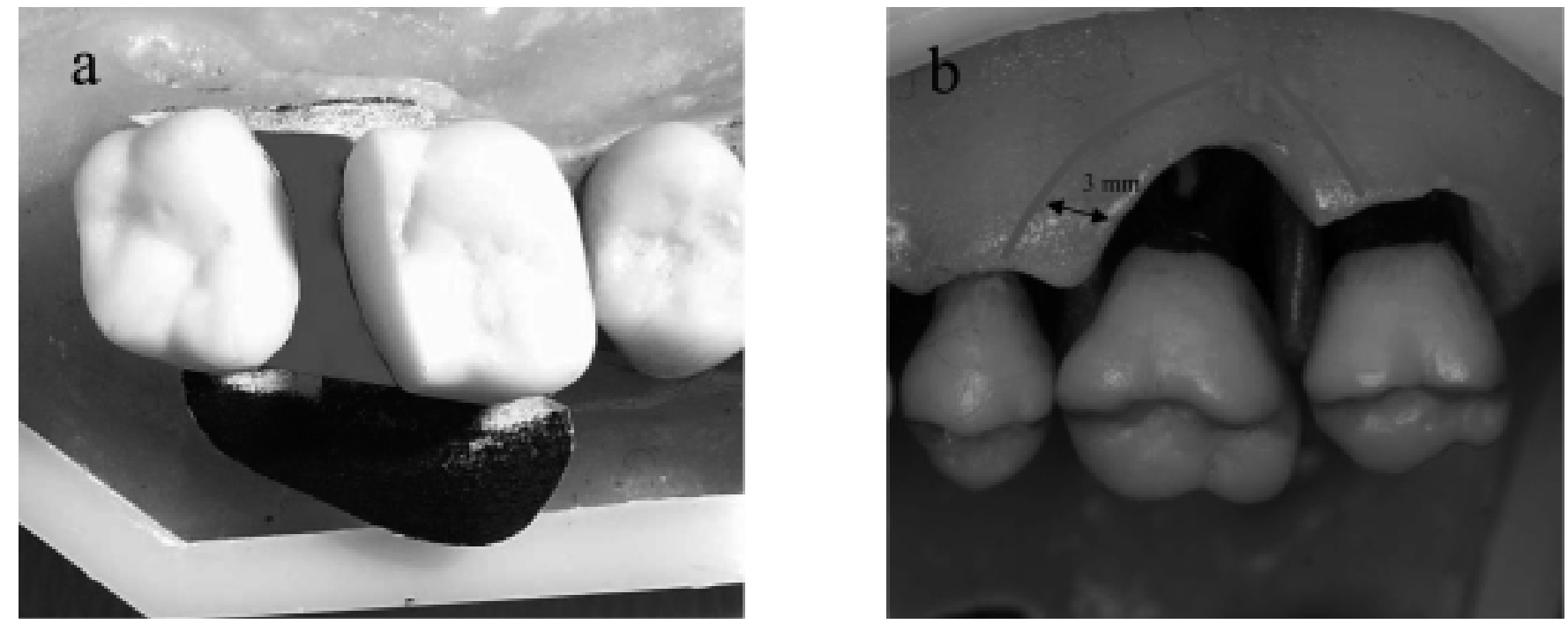

Fig. 3 (a) Measurement area at the interproximal site (black area). (b) The black outline indicates $3 \mathrm{~mm}$ from the defect.
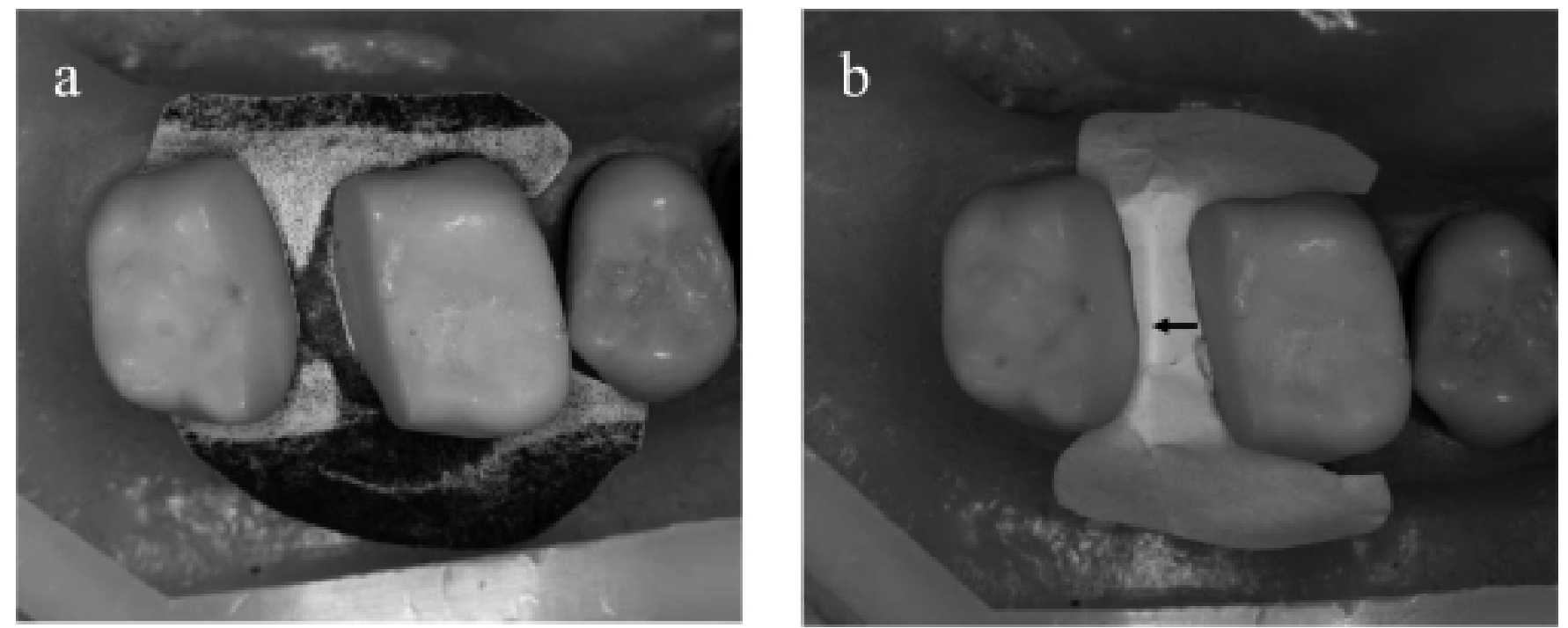

Fig. 4 (a) With the CT method, the membrane fitted well. (b) With the periapical method, the membrane became wrinkled.

part of the buccal site tended not to be covered by the membrane when the conventional method was employed (Figs. 6 and 7).

The CT method took $3.3 \pm 1.5 \mathrm{~min}$ (range 1.4-5.8), as compared to $6.2 \pm 3.0 \mathrm{~min}$ (range $7.5-10.6$ ) for the conventional method. Thus the CT method significantly reduced the time required to trim the membrane (Fig. 8).

\section{Clinical study}

Five otherwise healthy subjects (4 women and 1 man, age range $25-61$ years, mean age 50.2 years) with moderate to severe periodontal disease participated in this study. The patients had 2 (1 case) or 3 (5 cases) wall defects. Six teeth with periodontal attachment loss were used in this study. All the patients who participated signed an informed consent statement approved by the Ethics commitee of Nihon University school of Dentistry.

All of the patients underwent a thorough periodontal examination and initial treatment that included oral hygiene instruction, scaling, and root planing. Each patient was given comprehensive information concerning the nature, purpose, and potential risks of surgery. After induction of local anesthesia, sulcular incisions were made, and a fullthickness flap was raised in the area of the vertical defect to be treated. Thorough debridement and root preparation of the surgical site were carried out with ultrasonic and 
hand scalers.

GTR therapy was performed using an absorbable membrane (Bio Mend ${ }^{\circledR}$ ). Periapical radiographs were taken using a dental holder. An axial CT image based on the periapical radiograph was used to make the template, and we drew the outline of the membrane using PC drawing software (Fig. 9). The template was printed on transparency film, cut from the film, and sterilized before surgery (Fig. 10a). Using the template, the membrane could be cut easily using scissors, and the time required to adjust the GTR membrane was shortened by preparing the template

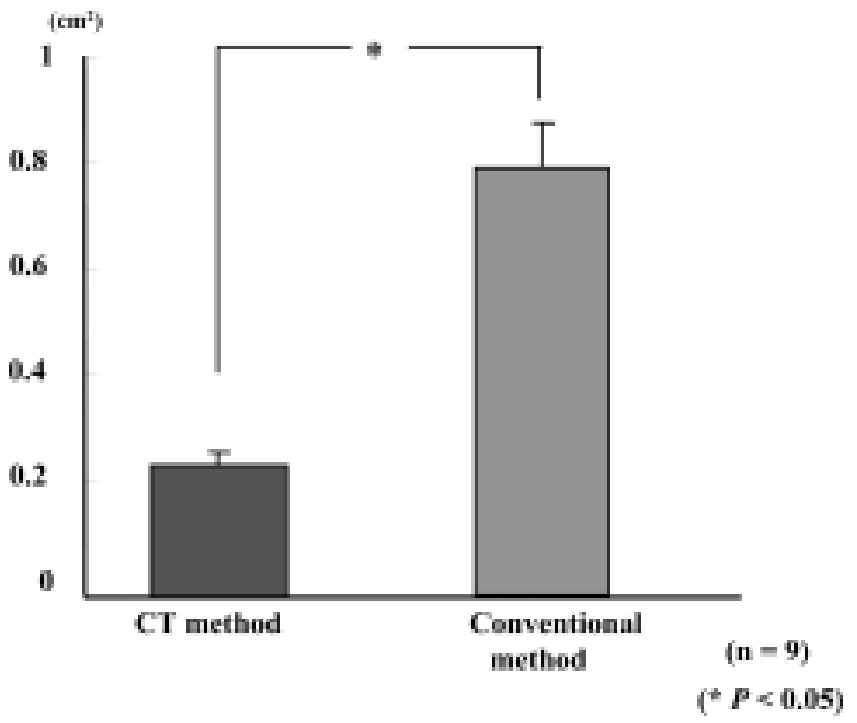

Fig. 5 Comparison of the area of the membrane at the interproximal site using the CT and periapical methods.

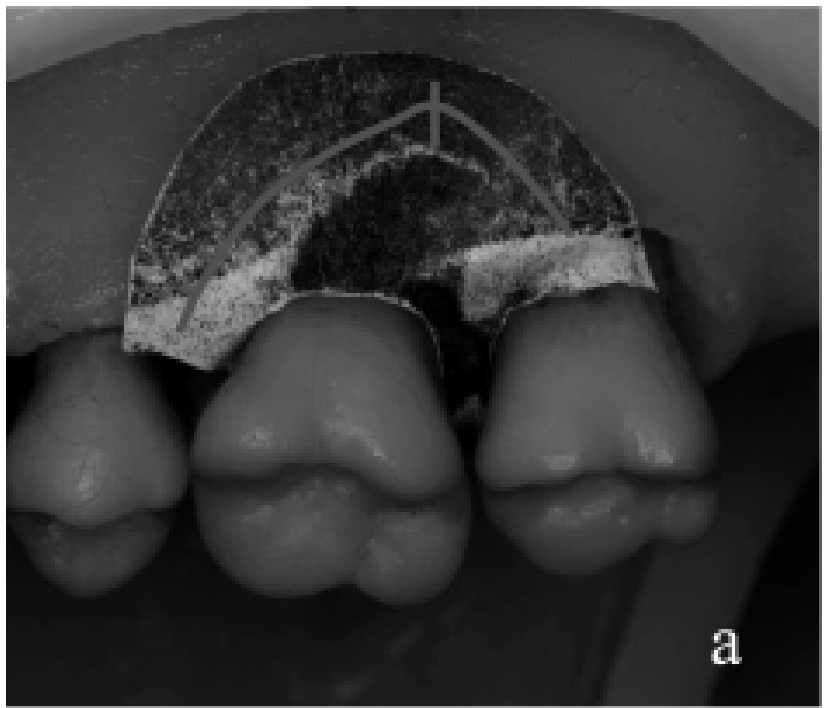

Fig. 6 (a) With the CT method, the membrane covered the defect completely. (b) With the periapical method, the membrane did not cover the defect completely at the mesial buccal site.

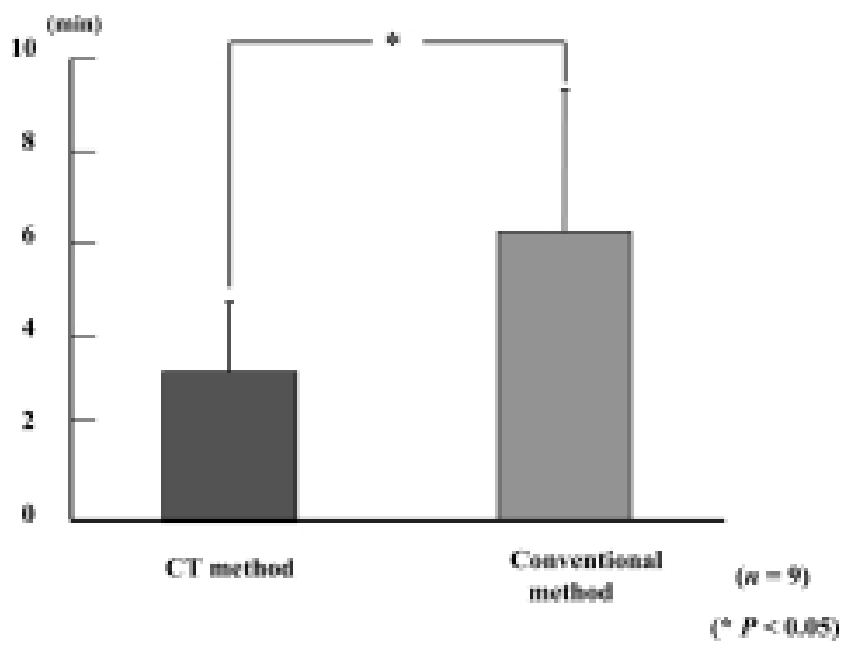

Fig. 8 A comparison of the time required for membrane trimming and adjustment using the CT and periapical methods.

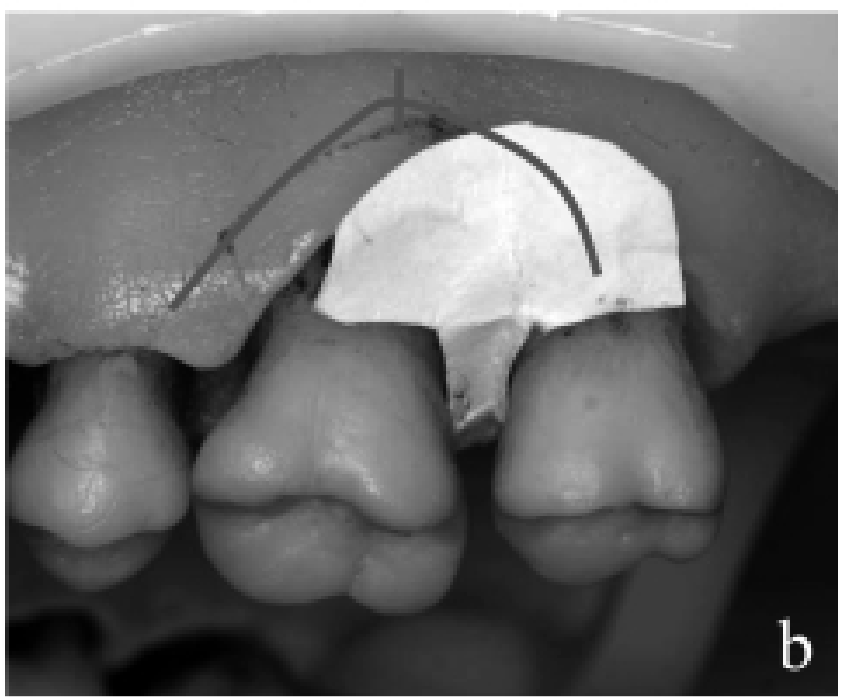



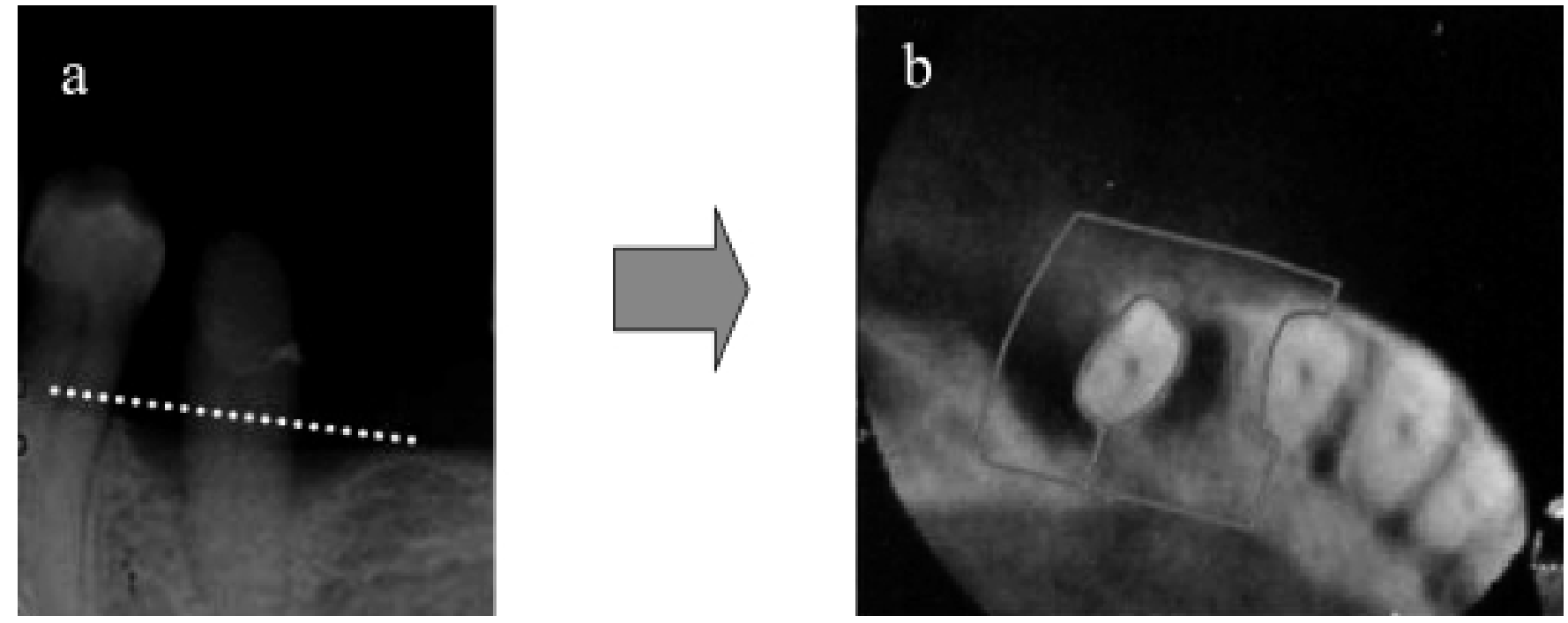

Fig. 9 (a) Periapical radiograph showing angular bone defects in \#34. (b) Axial CT images obtained in the plane of the periapical radiograph (dotted line). The solid line indicates the outline of the membrane.
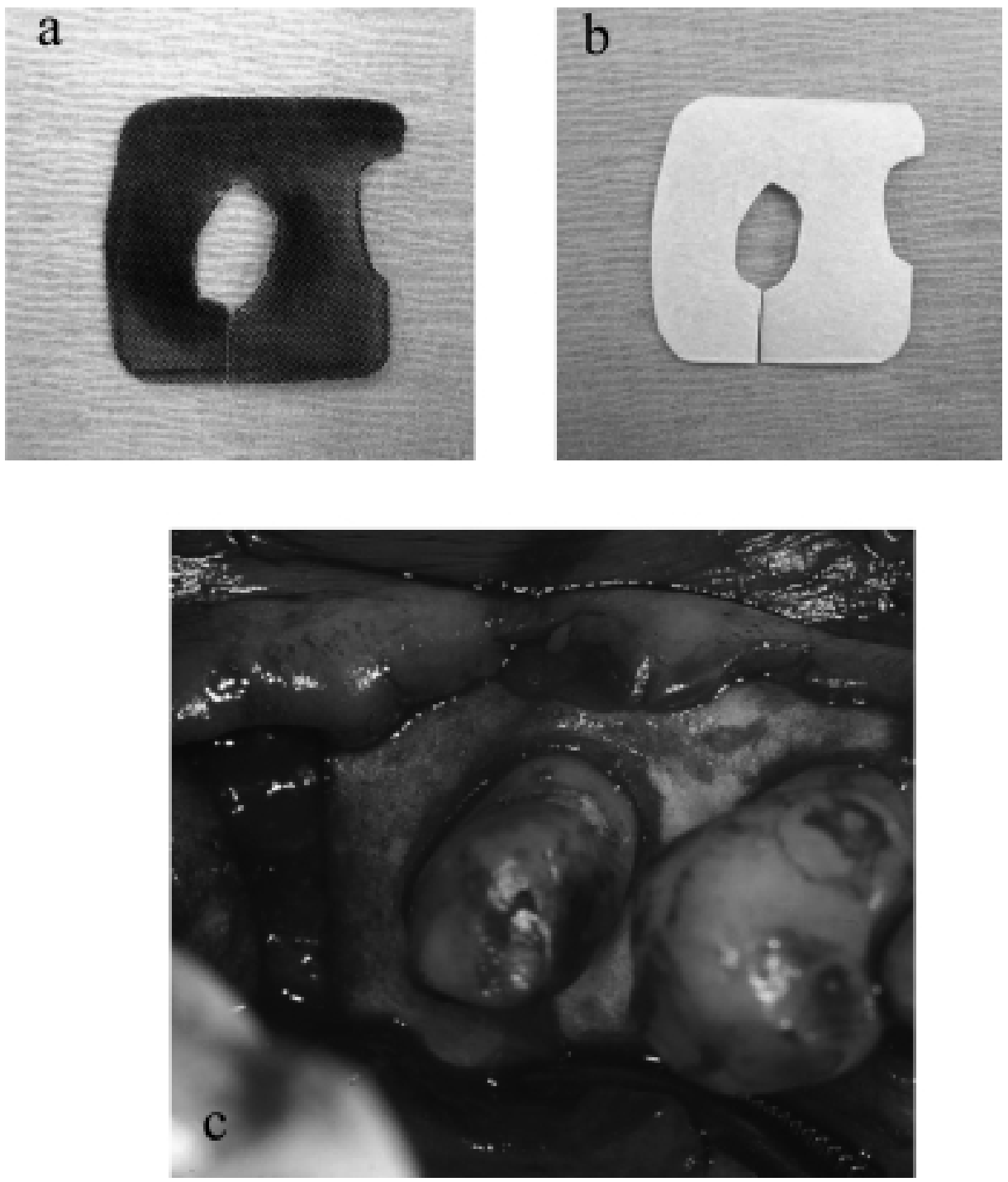

Fig. 10 (a) The outline of the membrane on transparency film as a template. (b) The membrane cut from the film. (c) Membrane adaptation during surgery. 

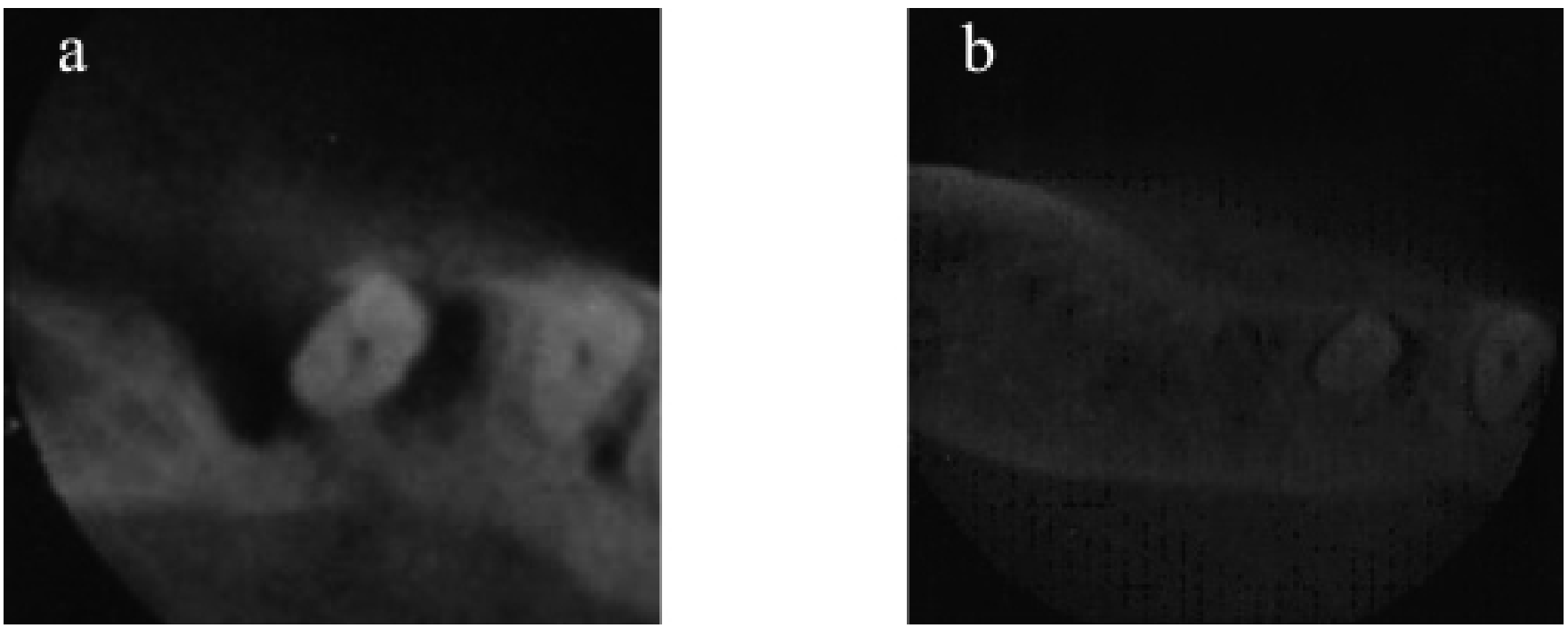

Fig. 11 (a) Axial CT image of \# 34 before surgery. (b) Improvement of the condition of the hard tissue after 18 months.

before surgery (Figs. 10b, c).

None of the sites exhibited membrane exposure, swelling, or suppuration at any time during the healing period. Six to 18 months after the operation, we took periapical radiographs and obtained $\mathrm{CT}$ images, and compared the hard tissue images on the CT images and periapical radiographs. The axial CT images clearly showed improvement of the condition of the hard tissue (Fig. 11).

\section{Discussion}

GTR procedures have been used to treat various periodontal defects (1-4), and the technical factors leading to success with GTR are careful flap design, correct membrane placement, good wound closure, and optimal postoperative plaque control. The barrier membrane must cover the defect and should extend beyond the defect border by 3 to $5 \mathrm{~mm}$ (12). Becker et al. (5) stated that to succeed with GTR therapy, clinicians must understand the defect anatomy and root trunk dimensions.

Absorbable membranes have been introduced in GTR to avoid a second operation for membrane removal, which not only benefits the patient but also eliminates any trauma that may occur to the immature, newly regenerated periodontal tissues during barrier removal. Premature removal of the membrane results in significantly decreased regeneration (13). However, absorbable membranes need to be trimmed to adapt them to the root and cover the bone defects. Trimming and adjusting a membrane to a complicated shape is difficult and prolongs surgery. Furthermore, if the membrane trimming fails, the monetary cost is much greater, as the membrane is expensive.

In such cases, we have used cone beam $\mathrm{CT}$ for diagnosis of angular bone defects. Accurate radiographs are needed in order for GTR therapy to succeed. Ito et al. (11) used cone beam CT to evaluate the outcome of regenerative therapy. The cone beam CT images allow observation of the morphology of periodontal bone defects in three dimensions because images can be obtained in any plane (sagittal, axial and coronal). We used only the axial image to make a template for the GTR membrane using transparency film, which can be sterilized before surgery. Further studies will be needed in order to adapt the membrane three-dimensionally.

This basic and clinical study confirmed that, using the template, the membrane can be easily trimmed and adapted to the root surface in either the maxillary or mandibular arch. In contrast, without the use of CT, our basic study showed that the membrane had a larger interproximal area, and was smaller at the mesial buccal site. Furthermore, the membrane trimming time was shown to be shortened using CT.

We observed a 4- to 6-mm clinical attachment gain and an improved condition of the hard tissues after 6 to 18 months, which may have been the result of optimal membrane adaptation and accurate diagnosis of bone defects using cone beam CT. Furthermore, no membrane exposure was encountered. Membrane exposure results in bacterial contamination $(14,15)$, which may impair successful regeneration. Ability to select the optimal shape and size of the membrane might decrease the chance of membrane exposure during the healing period.

According to the manufacturer's guidelines, the e-PTFE membrane technique should be used for the treatment of single-tooth defects or single interdental spaces, to allow 
optimal adaptation of the membrane. Kotschy et al. (16) used a large e-PTFE membrane to treat multiple bone defects, and observed a reduced probing depth and increased clinical attachment 36 months after surgery. An absorbable membrane can be used for multiple bone defects because the membrane can be trimmed to different sizes and shapes. Membrane adaptation is even more demanding when treating several adjacent teeth with multiple periodontal osseous defects and root irregularities, in which case trimming the membrane to a complicated shape is difficult. The present series included only one such case. Nevertheless, cone beam CT would be useful for the treatment of multiple bone defects.

In conclusion, there are a number of advantages with the use of cone beam CT for evaluating the morphology of periodontal bone defects and root proximity. We anticipate that this procedure will prove to be a useful aid to periodontal examination and diagnosis, and for predicting and evaluating the outcome of periodontal regenerative treatment.

\section{References}

1. Cortellini P, Pini Prato G, Tonetti MS (1993) Periodontal regeneration of human infrabony defects. 1. Clinical measures. J Periodontol 64, 254-260.

2. McClain PK, Schallhorn RG (1993) Long-term assessment of combined osseous composite grafting, root conditioning, and guided tissue regeneration. Int J Periodontics Restorative Dent 13, 9-27.

3. Becker W, Becker BE, Berg L, Prichard J, Caffesse R, Rosenberg E (1998) New attachment after treatment with root isolation procedure: report for treated Class III and Class II furcations and vertical osseous defects. Int J Periodontics Restorative Dent $8,8-23$.

4. Handelsman M, Davarpannah M, Celletti R (1991) Guided tissue regeneration with and without citric acid treatment in vertical osseous defects. Int $\mathrm{J}$ Periodontics Restorative Dent 11, 350-363.

5. Becker W, Becker BE (1993) Clinical application of guided tissue regeneration: surgical consideration. Periodonotol 2000 1, 46-53.

6. Ramachandran GN, Lakshminarayanan AV (1971) Three-dimensional reconstruction from radiographs and electron micrographs: application convolutions instead of Fourier Tranforms. Proc Natl Acad Sci U S A 68, 2236-2240.

7. Hounsfield GN (1973) Computerized transverse axial scanning (tomography). Part 1. Description system. Br J Radiol 46, 1016-1022.

8. Marsh JL, Vannier MW (1983) The "third" dimension in craniofacial surgery. Plast Reconstr Surg 71, 759-767.

9. Mutoh Y, Ohashi Y, Uchiyama N, Terada K, Hanada K, Sasaki F (1991) Three-dimensional analysis of condyler hyperplasia with computed tomogryphy. J Craniomaxillofac Surg 19, 49-55.

10. Vannier MW, Hildebolt CF, Conover G, Knapp RH, Yokoyama-Crothers N, Wang G (1997) Threedimensional dental imaging by spiral CT. A progress report. Oral Surg Oral Med Oral Pathol 84, 561-570.

11. Ito K, Yoshinuma N, Goke E, Arai Y, Shinoda K (2001) Clinical application of a new compact computed tomography system for evaluating the outcome of regenerative therapy: a case peport. J Periodontol 72, 696-702.

12. Quiñones CR, Cafesse RG (1995) Current status of guided periodontal tissue regeneration. Periodontol 2000 9, 55-68.

13. Lekholm U, Becker W, Dahlin C, Becker B, Donath K, Morrison E (1993) The role of early versus late removal of GTAM membranes on bone formation at oral implants placed into immediate extraction sockets. An experimentally study in dogs. Clin Oral Implants Res 4, 121-129.

14. Simion M, Baldoni M, Rossi P, Zaffe D (1994) A comparative study of the effectiveness of e-PTFE membranes with and without early exposure during the healing period. Int J Periodontics Restorative Dent 14, 167-180.

15. Nowzari H, Slots J (1994) Microorganisms in polytetrafluoroethylene barrier membranes for guided tissue regeneration. J Clin Periodontol 21, 203-210.

16. Kotschy P, Munzker R (1995) New dimensions in guided tissue regeneration treatment modalities for profound marginal periodontitis. Int J Periodontics Restorative Dent 15, 285-297. 